\title{
Correct and incorrect knowledge of the risk factors concerning the development of arterial hypertension. Part 2. Population of patients with identified arterial hypertension
}

\author{
Andrzej Kleinrok'1,2, Michał Kamiński', Tomasz Domański', Grażyna Prokop-Lewicka' \\ ${ }^{1}$ The Pope John Paul II Province Hospital, Zamość, Poland \\ ${ }^{2}$ University of Management and Administration in Zamość \\ Head of department: Andrzej Kleinrok
}

\begin{abstract}
Introduction. Hypertension (HT) and its complications significantly shorten the life expectancy in the world population. In addition to pharmacotherapy, life-style changes play a key role in treatment, which requires appropriate knowledge among patients.

Material and methods. The study was conducted in a group of 613 adults with HT. After obtaining sociodemographic data, performing anthropometric measurements, measuring resting blood pressure twice, the knowledge was assessed using a personally conducted questionnaire. The questions evaluated the effects of smoking, alcohol, coffee, salt, fat, irregular diet, low fibre and vegetable intake, as well as skimmed dairy products in diet, overweight and obesity, cholesterol, physical activity and stressful lifestyle on the development of HT.

Results. False knowledge of the influence on the development of HT in the case of alcohol drinking was found in 16\% of the respondents, coffee $-56.4 \%$, large amounts of salt $-7.2 \%$, large amounts of fats $-88 \%$, small amounts of fibre and vegetables and skimmed dairy products $-27.8 \%$, irregular diet $-46.4 \%$, cigarette smoking $-73.4 \%$, overweight and obesity $-4.4 \%$, high cholesterol $-86.9 \%$, low physical activity $-11.2 \% \%$, stressful lifestyle $-5.4 \%$ Conclusions. Incorrect knowledge of HT risk factors was most likely to be associated with excessive intake of fat, elevated cholesterol, smoking and coffee drinking. There were no common features of the people who answered the questions incorrectly. The results show the need to educate HT patients about the factors that aggravate this disease. key words: hypertension, risk factors, knowledge
\end{abstract}

Arterial Hypertens. 2017, vol. 21, no. 2, pages: 83-92 DOI: $10.5603 / A H .2017 .0011$

\section{Introduction}

Hypertension (HT) is a risk factor of many diseases, including renal failure, aortic dissection, stroke, ischaemic heart disease, significantly shortening life in the general population [24-26].
Raised blood pressure (RR), by increasing postload, causes left ventricular hypertrophy [27]. This results in a progressive restriction of wall motion leading to diastolic dysfunction. In addition, as a result of the compression of the hypopharynx capillaries

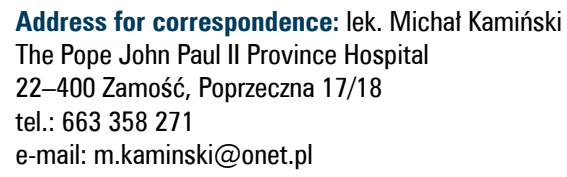


and the reduction of their density, the features of ischaemia appear. Left ventricular hypertrophy leads to left atrial enlargement, its remodelling and consequent supraventricular arrhythmias, including atrial fibrillation. In this type of arrhythmia, HT not only contributes to its formation but also raises the risk of ischaemic stroke in patients affected by this disorder. Reconstruction of the myocardium significantly increases the risk of ventricular arrhythmias, and consequently may lead to sudden cardiac death [28, 29].

HT leads to thickening of the intima and media of peripheral arteries, contributing to increased stiffness and atherothrombosis. This leads to the formation of ischaemic stroke in case of damage to carotid arteries and to acute coronary syndrome in case of coronary artery occlusion. Raised RR is also a major risk factor of aortic dissection [25, 29].

Kidneys are other organs that are damaged in the course of HT. Damage to renal artery endothelial cells leads to local inflammation, which results in gradual remodelling and fibrosis of the renal interstitium. Initially no clinical symptoms are observed, but over time the symptoms of chronic kidney disease become apparent [27, 29].

Raised RR is considered one of the main risk factors for both ischaemic stroke and haemorrhagic stroke. It is worth pointing out, however, that it also leads to clinically silent episodes of ischaemia resulting in dementia. In the case of the eye organ, arterial remodelling and bleeding that damage the retina occur, resulting in lower quality of life [27-29].

In order to prevent these complications, it is necessary to treat HT effectively, which, apart from pharmacotherapy, is based on lifestyle modification [27]. Therefore, it is so important for a patient to cooperate, which is inextricably linked to their knowledge of the factors that aggravate the disease.

The causes of HT development and, consequently, the increase in the demand for antihypertensive drugs include the consumption of alcohol, high amounts of sodium, small amounts of fibre, vegetables and skimmed dairy products, overweight and obesity, low physical activity and stressful lifestyle.

There were theoretical foundations to believe that some factors could affect the severity of HT, but no correlation was found in the study. This group includes cigarette smoking, coffee consumption, high fat intake, irregular diet, and lipid disorders.

With the aim of increasing the effectiveness of prevention it is helpful to know the group of patients with incorrect knowledge. Thus, it seemed interesting to assess the knowledge that HT patients have, and to find common characteristics of people with misconceptions. The aim of the study is estimate the HT patient's knowledge and extracting groups with incorrect information.

\section{Material and methods}

Data for the study were collected for the bachelor's degree thesis in the period from June to October 2012 in the Lublin Region by the students* of the Nursing Department of the Faculty of Physiotherapy and Pedagogy of the University of Management and Administration in Zamość. The study included an incidental group of 613 people, including 268 men, who reported to the GP practice due to HT. Each participant delivered a written consent to participate in the study. The scope and method of collecting data have been shown in Part 1.

To assess the knowledge the authorial survey questionnaire, approved by the Authorities and the Bioethics Committee of the University of Management and Administration in Zamość, was used. The data were collected personally by interviewers who asked about the effects of alcohol, coffee, large amounts of salt, large amounts of fat, small amounts of fibre and vegetables, and skimmed dairy products in the diet, irregular meals, smoking cigarettes and obesity, high cholesterol, insufficient physical activity, stressful lifestyle on development of HT.

The respondents could assess the impact of factors on HT by marking: high/moderate/low/no impact. They could also answer that they have no opinion. Responses were divided into two groups: high impact i.e. the combination of moderate and high impact responses and no impact, i.e. the combination of no and low impact responses.

The method of statistical analysis has been presented in Part 1.

\section{Results}

In the study group, the average age was 60.2 years, and women accounted for over 56\%. More than half of respondents lived in the city, more than $40 \%$ had basic or vocational education and almost three quarters were married. The average heart rate in the study group was 77.5 beats per minute, mean systolic blood pressure was $141 \mathrm{mmHg}$ and diastolic blood pressure was $84.7 \mathrm{mmHg}$. The remaining data have been shown in Table I.

Nearly $95 \%$ of the study population had either a poor knowledge or were unaware of the effect of 
Table I. General population characteristics ( $n=613$ )

\begin{tabular}{|l|c|}
\hline Sociodemographic data & Respondents \\
\hline Male gender & $268(43.7)$ \\
\hline Age (years) & $60.2(12.7)$ \\
\hline Residence - city & $316(51.5)$ \\
\hline Married & $457(74.5)$ \\
\hline Education secondary or higher & $350(57.1)$ \\
\hline Professionally active including: & $253(41.3)$ \\
\hline - intellectual work & $110(43.5)$ \\
\hline Number of people living in the household & $143(56.5)$ \\
\hline Monthly income/person (PLN) $\mathrm{n}=363^{*}$ & $2.7( \pm 1.6)$ \\
\hline Medical data & $1202.4( \pm 993.7)$ \\
\hline Current body weight [kg] & \\
\hline Current height [cm] & $81.8( \pm 15.1)$ \\
\hline Waist measurement [cm] & $167.5( \pm 9.6)$ \\
\hline - women [cm] & $98.1( \pm 15.4)$ \\
\hline - men [cm] & $96.1( \pm 16.4)$ \\
\hline Mean systolic RR [mmHg] & $100.7( \pm 13.6)$ \\
\hline Mean diastolic RR [mmHg] & $141.0( \pm 17.0)$ \\
\hline Average pulse rate (bpm) & $84.7( \pm 9.9)$ \\
\hline BMl & $77.5( \pm 23.1)$ \\
\hline - women & $29.2( \pm 6.6)$ \\
\hline - men & $28.8( \pm 4.8)$ \\
\hline Current smokers & $29.7( \pm 8.5)$ \\
\hline Current smokers and ex-smokers & $96(15.7)$ \\
\hline Average weekly consumption of pure alcohol [g] & $34.4( \pm 100.3)$ \\
\hline - women & $9.3( \pm 58.5)$ \\
\hline - men & $65.4( \pm 130.1)$ \\
\hline Data presented as n (\%) or mean $( \pm S D)$ & $(36.5)$ \\
\hline
\end{tabular}

Data presented as $n(\%)$ or mean $( \pm \mathrm{SD})$

$\mathrm{RR}$ — blood pressure; BMI — Body Mass Index; *number of respondents

high fat intake and high cholesterol on HT development. About $70 \%$ of the respondents had none or incorrect information about the impact of coffee consumption and irregular diet on HT, and as much as $86 \%$ about the influence of cigarette smoking on this disease. More than half of the respondents reported that eating small amounts of fibre, vegetables and skimmed dairy products did not affect HT, which is incompatible with current knowledge, or did not know about such a relationship, and less than one third gave a similar answer in case of alcohol. Of the respondents, $19 \%$ did not have a proper knowledge of the relationship between low physical activity and HT and almost $12 \%$ of the connection between high salt intake and HT. The respondents had best knowledge in the case of the influence of stressful lifestyle and overweight and obesity on the abovementioned disease. Here $9 \%$ and $7.3 \%$ respectively had no opinion or gave an incorrect answer (Table II).

Misconceptions about the effects of alcohol on the development of HT were reported in $16 \%$ of respondents. More often, people who did not receive information from the Internet (26\% vs. $16.6 \%$; $\mathrm{p}=0.019$ ) had incorrect information (Tables II and III).

Coffee, as a substance affecting HT, was indicated by $56.4 \%$ of respondents. This view was more frequently presented by people who received information from family and friends (70.9\% vs. $61.4 \%$; $\mathrm{p}=0.031)$ (Tables II and IV).

In the study group, $73.4 \%$ of people believed that cigarette smoking influences the development of HT, which is incompatible with current knowledge. Particularly, people who received information from health professionals $(86.5 \%$ vs. $76.6 \% ; p=0.007)$ and non-smokers ( 85.5 vs. $77.2 \mathrm{p}=0.045)$ had false knowledge (Tables II and V).

In the case of salt intake, $7.2 \%$ of the respondents believed that it did not influence the development of HT, which is incompatible with the current state of knowledge. Such opinion was slightly more frequent, at the edge of statistical significance, for those who did not receive information from health professionals $(11.1 \%$ vs. $6.3 \% ; \mathrm{p}=0.059)$ (Tables II and VI).

As many as $88 \%$ of respondents mistakenly answered the question about the effect of high intake of fat on the development of HT. Especially women $(96.1 \%$ vs. $92.1 \% ; \mathrm{p}=0.043)$ and people who achieved information from health professionals ( $96.1 \%$ vs. $89 \%$; $p=0.002$ ) had incorrect information (Tables II and VII).

Incorrect information about the influence of irregularly consumed meals on HT development was reported by $46.4 \%$ of the respondents. In the study group people acquiring information from health professionals $(64.3 \%$ vs. $50.0 \% ; \mathrm{p}=0.009)$, family and friends $(68.7 \%$ vs. $57.5 \%$; $=0.022)$ and the Internet ( $4 \%$ vs. $58.5 \% ; \mathrm{p}=0.042)$ were predominant. (Tables II and VIII).

In the study group, $27.8 \%$ of people had incorrect information about the effect of low fibre intake and vegetable consumption and skimmed dairy products on the development of HT. More often, people who did not achieve information from health professionals $(46.0 \%$ vs. $37.1 \% ; \mathrm{p}=0.099)$ had incorrect information. The above relationship was at the edge of statistical significance (Tables II and IX).

Overweight and obesity as a factor not affecting HT were indicated by $5.1 \%$ of respondents. More 
Table II. Patients' knowledge of factors affecting HT development $(n=613)$

\begin{tabular}{|l|c|c|c|c|c|}
\hline \multirow{2}{*}{ Patients' knowledge of factors affecting HT development } & \multicolumn{3}{|c|}{ Impact } & \multirow{2}{*}{ No opinion } \\
\cline { 2 - 5 } & High & Moderate & Low & No & \\
\hline Alcohol consumption & $266(43.4)$ & $157(25.6)$ & $84(13.7)$ & $14(2.3)$ & $92(15.0)$ \\
\hline Coffee consumption & $145(23.6)$ & $201(32.8)$ & $144(23.5)$ & $47(7.7)$ & $76(12.4)$ \\
\hline Smoking & $318(51.9)$ & $132(21.5)$ & $76(12.4)$ & $9(1.5)$ & $78(12.7)$ \\
\hline Consuming large amount of salt & $426(69.5)$ & $116(18.9)$ & $35(5.7)$ & $9(1.5)$ & $27(4.4)$ \\
\hline Consuming large amount of fat & $402(65.6)$ & $137(22.4)$ & $22(3.6)$ & $10(1.6)$ & $42(6.8)$ \\
\hline Irregular diet & $124(20.3)$ & $160(26.1)$ & $141(23.0)$ & $40(6.5)$ & $148(24.1)$ \\
\hline $\begin{array}{l}\text { Consuming a small amount of fibre, vegetables and skimmed } \\
\text { dairy products }\end{array}$ & $128(20.9)$ & $134(21.8)$ & $112(18.3)$ & $58(9.5)$ & $181(29.5)$ \\
\hline Overweight and obesity & $499(81.4)$ & $69(11.3)$ & $23(3.8)$ & $4(0.6)$ & $18(2.9)$ \\
\hline High level of cholesterol & $444(72.4)$ & $89(14.5)$ & $29(4.8)$ & $5(0.8)$ & $46(7.5)$ \\
\hline Low physical activity & $343(56.0)$ & $153(25.0)$ & $53(8.6)$ & $16(2.6)$ & $48(7.8)$ \\
\hline Stressful lifestyle & $457(74.5)$ & $101(16.5)$ & $25(4.1)$ & $8(1.3)$ & $22(3.6)$ \\
\hline
\end{tabular}

Data presented as $\mathrm{n}(\%)$

Table III. Factors affecting knowledge of the influence of alcohol on the development of HT

\begin{tabular}{|l|c|c|c|}
\hline $\mathbf{n}=\mathbf{5 2 1 *}$ & Significant impact** & No impact*** & $\mathbf{p}$ \\
\hline Female/male & $245(81.4) / 178(80.9)^{* *}$ & $56(18.6) / 42(19.1)$ & $\mathrm{p}=0.888$ \\
\hline Age $<49$ years/> 49 years & $93(86.1) / 330(79.9)$ & $15(13.9) / 83(20.1)$ & $\mathrm{P}=0.142$ \\
\hline Married/single & $321(81.3) / 102(80.9)$ & $74(18.7) / 24(19.1)$ & $\mathrm{p}=0.940$ \\
\hline Rural/urban residence & $199(82.9) / 224(79.7)$ & $41(17.1) / 57(20.3)$ & $\mathrm{p}=0.351$ \\
\hline Primary education or vocational/secondary or higher & $181(84.6) / 242(78.8)$ & $33(15.4) / 65(21.2)$ & $\mathrm{p}=0.098$ \\
\hline Professionally active yes/no & $182(80.5) / 241(81.7)$ & $44(19.5) / 54(18.3)$ & $\mathrm{p}=0.736$ \\
\hline Knowledge from health professionals yes/no & $325(81.7) / 98(79.7)$ & $73(18.3) / 25(20.3)$ & $\mathrm{p}=0.623$ \\
\hline Knowledge from the press, TV and newspapers, books yes/no & $273(79.8) / 150(83.8)$ & $69(20.2) / 29(16.2)$ & $\mathrm{p}=0.270$ \\
\hline Knowledge from family and friends yes/no & $143(84.6) / 280(79.5)$ & $26(15.4) / 72(20.5)$ & $\mathrm{p}=0.166$ \\
\hline Knowledge from the Internet yes/no & $91(74.0) / 332(83.4)$ & $32(26.0) / 66(16.6)$ & $\mathrm{p}=0.019$ \\
\hline Obesity no/yes & $268(80.1) / 155(81.6)$ & $63(19.0) / 35(18.4)$ & $\mathrm{p}=0.860$ \\
\hline Current smoker yes/no & $66(79.5) / 357(81.5)$ & $17(20.5) / 81(18.5)$ & $\mathrm{p}=0.670$ \\
\hline
\end{tabular}

Data is presented as $n(\%) ; ~ *$ not including people who replied "I have no opinion"; ** combination of high and moderate impact; ${ }^{* * *}$ combination of low and no impact

Table IV. Factors affecting knowledge of the influence of coffee on the development of HT

\begin{tabular}{|l|c|c|c|}
\hline $\mathbf{n}=\mathbf{5 3 7 *}$ & Significant impact ** & No impact*** & $\mathbf{p}$ \\
\hline Female/male & $200(64.1) / 146(64.9)$ & $112(35.9) / 79(35.1)$ & $\mathrm{p}=0.850$ \\
\hline Age $<49$ years/> 49 years & $78(68.4) / 268(63.4)$ & $36(31.6) / 155(36.6)$ & $\mathrm{p}=0.320$ \\
\hline Married/single & $261(65.6) / 85(61.1)$ & $137(34.4) / 54(38.9)$ & $\mathrm{p}=0.348$ \\
\hline Rural/urban residence & $164(65.3) / 182(63.6)$ & $87(34.7) / 104(36.4)$ & $\mathrm{p}=0.681$ \\
\hline Primary education or vocational/secondary or higher & $146(65.8) / 200(63.5)$ & $76(34.2) / 115(36.5)$ & $\mathrm{p}=0.588$ \\
\hline Professionally active yes/no & $155(68.0) / 191(61.8)$ & $73(32.0) / 118(38.2)$ & $\mathrm{p}=0.140$ \\
\hline Knowledge from health professionals yes/no & $273(66.6) / 73(57.5)$ & $137(33.4) / 54(43.5)$ & $\mathrm{p}=0.061$ \\
\hline Knowledge from the press, TV and newspapers, books yes/no & $220(62.7) / 126(67.7)$ & $131(37.3) / 60(32.3)$ & $\mathrm{p}=0.243$ \\
\hline Knowledge from family and friends yes/no & $122(70.9) / 224(61.4)$ & $50(29.1) / 141(38.6)$ & $\mathrm{p}=0.031$ \\
\hline Knowledge from the Internet yes/no & $80(64.0) / 266(64.6)$ & $45(36.0) / 146(35.4)$ & $\mathrm{p}=0.908$ \\
\hline Obesity no/yes & $220(65.9) / 126(62.1)$ & $114(34.1) / 77(37.9)$ & $\mathrm{p}=0.373$ \\
\hline Current smoker yes/no & $53(60.9) / 293(65.1)$ & $34(39.1) / 157(34.9)$ & $\mathrm{p}=0.455$ \\
\hline
\end{tabular}

Data is presented as $n(\%) ;{ }^{*}$ not including people who replied "I have no opinion"; ** combination of high and moderate impact; ***combination of low and no impact 
Table V. Factors affecting knowledge of the influence of cigarette smoking on the development of HT

\begin{tabular}{|l|c|c|c|}
\hline $\mathbf{n}=\mathbf{5 3 5 *}$ & Significant impact** & No impact*** & $\mathbf{p}$ \\
\hline Female/male & $255(84.7) / 195(83.3)$ & $46(15.3) / 39(16.7)$ & $\mathrm{p}=0.664$ \\
\hline Age $<49$ years/> 49 years & $94(82.5) / 356(84.6)$ & $20(17.5) / 65(15.4)$ & $\mathrm{p}=0.586$ \\
\hline Married/single & $336(83.8) / 114(85.0)$ & $65(16.2) / 20(14.9)$ & $\mathrm{p}=0.725$ \\
\hline Rural/urban residence & $212(85.1) / 238(83.2)$ & $37(14.9) / 48(16.8)$ & $\mathrm{P}=0.544$ \\
\hline Primary education or vocational/secondary or higher & $189(85.5) / 261(83.1)$ & $32(14.5) / 53(16.9)$ & $\mathrm{p}=0.455$ \\
\hline Professionally active yes/no & $188(81.7) / 262(85.9)$ & $42(18.3) / 43(14.1)$ & $\mathrm{p}=0.192$ \\
\hline Knowledge from health professionals yes/no & $352(86.5) / 98(76.6)$ & $55(13.5) / 30(23.4)$ & $\mathrm{p}=0.007$ \\
\hline Knowledge from the press, TV and newspapers, books yes/no & $292(84.9) / 158(82.7)$ & $52(15.1) / 33(17.3)$ & $\mathrm{p}=0.512$ \\
\hline Knowledge from family and friends yes/no & $148(8.6) / 302(82.5)$ & $21(12.4) / 64(17.5)$ & $\mathrm{p}=0.137$ \\
\hline Knowledge from the Internet yes/no & $101(81.4) / 349(84.9)$ & $23(18.6) / 62(15.1)$ & $\mathrm{p}=0.355$ \\
\hline Obesity no/yes & $274(82.5) / 176(86.7)$ & $58(17.5) / 27(13.3)$ & $\mathrm{p}=0.201$ \\
\hline Current smoker yes/no & $71(77.2) / 379(85.5)$ & $21(22.8) / 64(14.5)$ & $\mathrm{p}=0.045$ \\
\hline
\end{tabular}

Data is presented as n (\%); *not including people who replied "I have no opinion"; *** combination of high and moderate impact; ${ }^{* * *}$ combination of low and no impact

Table VI. Factors affecting knowledge of the influence of large amounts of salt on the development of HT

\begin{tabular}{|l|c|c|c|}
\hline $\mathbf{n}=\mathbf{5 8 6 *}$ & Significant impact** & No impact*** & $\mathbf{p}$ \\
\hline Female/male & $314(93.4) / 228(91.2)$ & $22(6.6) / 22(8.8)$ & $\mathrm{p}=0.306$ \\
\hline Age $<49$ years/> 49 years & $111(94.1) / 431(92.1)$ & $7(5.9) / 37(7.9)$ & $\mathrm{P}=0.467$ \\
\hline Married/single & $404(92.9) / 138(91.4)$ & $31(7.1) / 13(8.6)$ & $\mathrm{p}=0.551$ \\
\hline Rural/urban residence & $264(93.3) / 278(91.7)$ & $19(6.7) / 25(8.3)$ & $\mathrm{p}=0.480$ \\
\hline Primary education or vocational/secondary or higher & $232(93.1) / 310(92.0)$ & $17(6.8) / 27(8.0)$ & $\mathrm{p}=0.591$ \\
\hline Professionally active yes/no & $228(93.1) / 314(92.1)$ & $17(6.9) / 27(7.9)$ & $\mathrm{p}=0.657$ \\
\hline Knowledge from health professionals yes/no & $414(93.7) / 128(88.9)$ & $28(6.3) / 16(11.1)$ & $\mathrm{p}=0.059$ \\
\hline Knowledge from the press, TV and newspapers, books yes/no & $355(93.7) / 187(90.3)$ & $24(6.3) / 20(9.7)$ & $\mathrm{p}=0.144$ \\
\hline Knowledge from family and friends yes/no & $176(92.1) / 366(92.7)$ & $15(7.9) / 29(7.3)$ & $\mathrm{p}=0.826$ \\
\hline Knowledge from the Internet yes/no & $125(94.7) / 417(91.8)$ & $7(5.3) / 37(8.2)$ & $\mathrm{p}=0.275$ \\
\hline Obesity no/yes & $330(92.2) / 212(93.0)$ & $28(7.8) / 16(7.0)$ & $\mathrm{p}=0.719$ \\
\hline Current smoker yes/no & $82(89.1) / 460(93.1)$ & $10(10.9) / 34(6.9)$ & $\mathrm{p}=0.183$ \\
\hline
\end{tabular}

Data is presented as $\mathrm{n}(\%) ;$ * ${ }^{*}$ ot including people who replied "I have no opinion"; ${ }^{* *}$ combination of high and moderate impact; ${ }^{* *}$ combination of low and no impact

Table VII. Factors affecting knowledge of the influence of large amounts of fat on the development of HT

\begin{tabular}{|l|c|c|c|}
\hline $\mathbf{n}=\mathbf{5 7 1 *}$ & Significant impact** & No impact*** & $\mathbf{p}$ \\
\hline Female/male & $317(96.1) / 222(92.1)$ & $13(3.9) / 19(7.9)$ & $\mathrm{p}=0.043$ \\
\hline Age $<49$ years/> 49 years & $112(95.7) / 427(94.0)$ & $5(4.3) / 27(6.0)$ & $\mathrm{p}=0.483$ \\
\hline Married/single & $403(96.2) / 136(95.1)$ & $25(5.8) / 7(4.9)$ & $\mathrm{p}=0.670$ \\
\hline Rural/urban residence & $255(94.1) / 284(94.7)$ & $16(5.9) / 16(5.3)$ & $\mathrm{p}=0.767$ \\
\hline Primary education or vocational/secondary or higher & $224(93.3) / 315(95.2)$ & $16(6.7) / 16(4.8)$ & $\mathrm{p}=0.347$ \\
\hline Professionally active yes/no & $229(95.0) / 310(93.9)$ & $12(5.0) / 20(6.1)$ & $\mathrm{p}=0.579$ \\
\hline Knowledge from health professionals yes/no & $418(96.1) / 121(89.0)$ & $17(3.9) / 15(11.0)$ & $\mathrm{p}=0.002$ \\
\hline Knowledge from the press, TV and newspapers, books yes/no & $352(94.4) / 187(94.4)$ & $21(5.6) / 11(5.6)$ & $\mathrm{p}=0.971$ \\
\hline Knowledge from family and friends yes/no & $174(93.0) / 365(95.0)$ & $13(7.0) / 19(5.0)$ & $\mathrm{p}=0.329$ \\
\hline Knowledge from the Internet yes/no & $128(97.0) / 411(93.6)$ & $4(3.0) / 28(6.4)$ & $\mathrm{p}=0.143$ \\
\hline Obesity no/yes & $326(93.4) / 213(95.9)$ & $23(6.6) / 9(4.1)$ & $\mathrm{p}=0.199$ \\
\hline Current smoker yes/no & $83(96.5) / 456(94.0)$ & $3(3.5) / 29(6.0)$ & $\mathrm{p}=0.355$ \\
\hline
\end{tabular}

Data is presented as $\mathrm{n}(\%) ;{ }^{*}$ not including people who replied "I have no opinion"; ${ }^{*}$ combination of high and moderate impact; ${ }^{* * *}$ combination of low and no impact 
Table VIII. Factors affecting knowledge of the influence of irregular diet on the development of HT

\begin{tabular}{|l|c|c|c|}
\hline $\mathbf{n}=465^{*}$ & Significant impact** & No impact*** & $\mathbf{p}$ \\
\hline Female/male & $164(62.8) / 120(58.8)$ & $97(37.2) / 84(41.2)$ & $\mathrm{p}=0.379$ \\
\hline Age $<49$ years/> 49 years & $71(68.9) / 213(58.8)$ & $32(31.1) / 149(41.2)$ & $\mathrm{p}=0.064$ \\
\hline Married/single & $210(60.0) / 74(64.3)$ & $140(40.0) / 41(35.7)$ & $\mathrm{p}=0.407$ \\
\hline Rural/urban residence & $124(57.7) / 160(64.0)$ & $91(42.3) / 90(36.0)$ & $\mathrm{p}=0.163$ \\
\hline Primary education or vocational/secondary or higher & $107(56.3) / 177(64.4)$ & $83(43.7) / 98(35.6)$ & $\mathrm{p}=0.080$ \\
\hline Professionally active yes/no & $134(66.0) / 150(57.2)$ & $69(34.0) / 112(42.8)$ & $\mathrm{p}=0.055$ \\
\hline Knowledge from health professionals yes/no & $232(64.3) / 52(50.0)$ & $129(35.7) / 52(50.0)$ & $\mathrm{p}=0.009$ \\
\hline Knowledge from the press, TV and newspapers, books yes/no & $177(60.2) / 107(62.6)$ & $117(39.8) / 64(37.4)$ & $\mathrm{p}=0.613$ \\
\hline Knowledge from family and friends yes/no & $101(68.7) / 183(57.5)$ & $46(31.3) / 135(42.5)$ & $\mathrm{p}=0.022$ \\
\hline Knowledge from the Internet yes/no & $75(69.4) / 209(58.5)$ & $33(30.6) / 148(41.5)$ & $\mathrm{p}=0.042$ \\
\hline Obesity no/yes & $170(60.1) / 114(62.6)$ & $113(39.9) / 68(37.4)$ & $\mathrm{p}=0.580$ \\
\hline Current smoker yes/no & $46(58.2) / 238(61.7)$ & $33(41.8) / 148(38.3)$ & $\mathrm{p}=0.569$ \\
\hline
\end{tabular}

Data is presented as n (\%); *not including people who replied "I have no opinion"; *** combination of high and moderate impact; ${ }^{* * *}$ combination of low and no impact

Table IX. Factors affecting knowledge of the influence of small amounts of fibre and vegetables and skimmed dairy products on the development of HT

\begin{tabular}{|l|c|c|c|}
\hline $\mathbf{n}=432^{*}$ & Significant impact** & No impact*** & $\mathbf{p}$ \\
\hline Female/male & $166(63.1) / 96(56.8)$ & $97(36.9) / 73(43.2)$ & $\mathrm{p}=0.190$ \\
\hline Age $<49$ years/> 49 years & $55(63.9) / 207(59.8)$ & $31(36.1) / 139(40.2)$ & $\mathrm{p}=0.483$ \\
\hline Married/single & $193(59.6) / 69(63.9)$ & $131(40.4) / 39(36.1)$ & $\mathrm{p}=0.426$ \\
\hline Rural/urban residence & $120(59.1) / 142(62.0)$ & $83(40.9) / 87(38.0)$ & $\mathrm{p}=0.539$ \\
\hline Primary education or vocational/secondary or higher & $100(60.6) / 162(60.7)$ & $65(39.4) / 105(39.3)$ & $\mathrm{p}=0.989$ \\
\hline Professionally active yes/no & $121(61.4) / 141(60.0)$ & $76(38.6) / 94(40.0)$ & $\mathrm{p}=0.763$ \\
\hline Knowledge from health professionals yes/no & $202(62.9) / 60(54.0)$ & $119(37.1) / 51(46.0)$ & $\mathrm{p}=0.099$ \\
\hline Knowledge from the press, TV and newspapers, books yes/no & $184(62.8) / 78(56.1)$ & $109(37.2) / 61(43.9)$ & $\mathrm{p}=0.184$ \\
\hline Knowledge from family and friends yes/no & $87(64.9) / 175(58.7)$ & $47(35.1) / 123(41.3)$ & $\mathrm{p}=0.222$ \\
\hline Knowledge from the Internet yes/no & $64(58.7) / 198(61.3)$ & $45(41.3) / 125(38.7)$ & $\mathrm{p}=0.633$ \\
\hline Obesity no/yes & $148(58.0) / 114(64.4)$ & $107(42.0) / 63(35.6)$ & $\mathrm{p}=0.183$ \\
\hline Current smoker yes/no & $36(52.9) / 226(62.1)$ & $32(47.1) / 138(37.9)$ & $\mathrm{p}=0.156$ \\
\hline
\end{tabular}

Data is presented as $\mathrm{n}(\%) ;{ }^{*}$ not including people who replied "I have no opinion"; ${ }^{*}$ combination of high and moderate impact; ${ }^{* * *}$ combination of low and no impact

often, people living in rural areas $(6.6 \%$ vs. $2.6 \%$; $\mathrm{p}=0.019$ ) had incorrect information (Tables II and X).

In the study population, as many as $86.9 \%$ of the respondents considered high cholesterol to have an impact on the development of HT. People who received information from family and friends $(97.2 \%$ vs. $92.5 \% ; \mathrm{p}=0.028)$ were more likely to have incorrect information (Tables II and XI).

In the case of low physical activity, $11.2 \%$ of the respondents stated that it did not influence the development of HT. Especially the rural population ( $15.6 \%$ vs. $9.1 \% ; \mathrm{p}=0.019)$, people with basic or vocational education $(16.7 \%$ vs. $9.1 \% ; \mathrm{p}=0.007)$, those not receiving information from health profes- sionals (17.7 vs. $10.4 \% ; \mathrm{p}=0.021)$ had incorrect information (Tables II and XII).

Among the respondents, $5.4 \%$ had a misconception about the impact of stressful lifestyle on the $\mathrm{HT}$, and this situation was more common among men $(7.8 \%$ vs. $3.9 \% ; \mathrm{p}=0.041)$ and those who did not receive information from health professionals $(10.6 \% \%$ vs. $3.9 \% ; \mathrm{p}=0.002)$ (Tables II and XIII).

\section{Discussion}

Effective HT treatment includes not only pharmacotherapy, but also involving the patient in the elim- 
Table X. Factors affecting knowledge of the influence of overweight and obesity on the development of HT

\begin{tabular}{|l|c|c|c|}
\hline $\mathbf{n}=\mathbf{5 9 5 *}$ & Significant impact $^{* *}$ & No impact*** & $\mathbf{p}$ \\
\hline Female/male & $326(96.4) / 242(94.2)$ & $12(3.6) / 15(5.8)$ & $\mathrm{p}=0.184$ \\
\hline Age $<49$ years/> 49 years & $114(95.8) / 454(95.4)$ & $5(4.2) / 22(4.6)$ & $\mathrm{p}=0.844$ \\
\hline Married/single & $423(95.1) / 145(96.7)$ & $22(4.9) / 5(3.3)$ & $\mathrm{p}=0.412$ \\
\hline Rural/urban residence & $269(93.4) / 299(97.4)$ & $19(6.6) / 8(2.6)$ & $\mathrm{p}=0.019$ \\
\hline Primary education or vocational/secondary or higher & $239(94.5) / 329(96.2)$ & $14(5.5) / 13(3.8)$ & $\mathrm{p}=0.315$ \\
\hline Professionally active yes/no & $237(95.6) / 331(95.4)$ & $11(4.4) / 16(4.6)$ & $\mathrm{p}=0.919$ \\
\hline Knowledge from health professionals yes/no & $431(96.4) / 137(92.6)$ & $16(3.6) / 11(7.4)$ & $\mathrm{p}=0.051$ \\
\hline Knowledge from the press, TV and newspapers, books yes/no & $370(96.1) / 198(94.3)$ & $15(3.9) / 12(5.7)$ & $\mathrm{p}=0.309$ \\
\hline Knowledge from family and friends yes/no & $184(95.3) / 384(95.5)$ & $9(4.7) / 18(4.5)$ & $\mathrm{p}=0.919$ \\
\hline Knowledge from the Internet yes/no & $129(97.0) / 439(95.0)$ & $4(3.0) / 23(5.0)$ & $\mathrm{p}=0.336$ \\
\hline Obesity no/yes & $340(94.7) / 228(96.6)$ & $19(5.3) / 8(3.4)$ & $\mathrm{p}=0.275$ \\
\hline Current smoker yes/no & $86(93.5) / 482(95.8)$ & $6(6.5) / 21(4.2)$ & $\mathrm{p}=0.320$ \\
\hline
\end{tabular}

Data is presented as n (\%); *not including people who replied "I have no opinion"; *** combination of high and moderate impact; ${ }^{* * *}$ combination of low and no impact

Table XI. Factors affecting knowledge of the influence of high cholesterol on the development of HT

\begin{tabular}{|l|c|c|c|}
\hline $\mathbf{n}=\mathbf{5 6 7 *}$ & Significant impact** & No impact*** & $\mathbf{p}$ \\
\hline Female/male & $311(94.5) / 222(93.3)$ & $18(5.5) / 16(6.7)$ & $\mathrm{p}=0.536$ \\
\hline Age $<49$ years/> 49 years & $109(93.2) / 424(94.2)$ & $8(6.8) / 26(5.8)$ & $\mathrm{p}=0.667$ \\
\hline Married/single & $402(93.9) / 131(94.2)$ & $26(6.1) / 8(5.8)$ & $\mathrm{p}=0.890$ \\
\hline Rural/urban residence & $255(94.1) / 278(93.9)$ & $16(5.9) / 18(6.1)$ & $\mathrm{P}=0.929$ \\
\hline Primary education or vocational/secondary or higher & $219(92.8) / 314(94.9)$ & $17(7.2) / 17(5.1)$ & $\mathrm{p}=0.307$ \\
\hline Professionally active yes/no & $220(93.2) / 313(94.6)$ & $16(6.8) / 18(5.4)$ & $\mathrm{p}=0.507$ \\
\hline Knowledge from health professionals yes/no & $408(94.7) / 125(91.9)$ & $23(5.3) / 11(8.1)$ & $\mathrm{p}=0.239$ \\
\hline Knowledge from the press, TV and newspapers, books yes/no & $345(94.0) / 188(94.0)$ & $22(6.0) / 12(6.0)$ & $\mathrm{p}=0.998$ \\
\hline Knowledge from family and friends yes/no & $175(97.2) / 358(92.5)$ & $5(2.8) / 29(7.5)$ & $\mathrm{p}=0.028$ \\
\hline Knowledge from the Internet yes/no & $122(94.6) / 411(93.8)$ & $7(5.4) / 27(6.2)$ & $\mathrm{p}=0.756$ \\
\hline Obesity no/yes & $325(93.7) / 208(94.5)$ & $22(6.3) / 12(5.5)$ & $\mathrm{p}=0.665$ \\
\hline Current smoker yes/no & $80(89.9) / 453(94.8)$ & $9(10.1) / 25(5.2)$ & $\mathrm{p}=0.075$ \\
\hline
\end{tabular}

Data is presented as $\mathrm{n}(\%) ;{ }^{*}$ not including people who replied "I have no opinion"; ** combination of high and moderate impact; ***combination of low and no impact

Table XII. Factors affecting knowledge of the influence of low physical activity on the development of HT

\begin{tabular}{|l|c|c|c|}
\hline $\mathbf{n}=\mathbf{5 6 5 *}$ & Significant impact** $^{*}$ & No impact*** & $\mathbf{p}$ \\
\hline Female/male & $283(87.9) / 213(87.6)$ & $39(12.1) / 30(12.4)$ & $\mathrm{p}=0.933$ \\
\hline Age $<49$ years/> 49 years & $104(89.7) / 392(87.3)$ & $12(10.3) / 57(12.7)$ & $\mathrm{p}=0.491$ \\
\hline Married/single & $381(89.2) / 115(83.3)$ & $46(10.8) / 23(16.7)$ & $\mathrm{p}=0.066$ \\
\hline Rural/urban residence & $227(84.4) / 269(90.9)$ & $42(15.6) / 27(9.1)$ & $\mathrm{p}=0.019$ \\
\hline Primary education or vocational/secondary or higher & $195(83.3) / 301(90.9)$ & $39(16.7) / 30(9.1)$ & $\mathrm{p}=0.007$ \\
\hline Professionally active yes/no & $211(87.5) / 285(88.0)$ & $30(12.5) / 39(12.0)$ & $\mathrm{p}=0.883$ \\
\hline Knowledge from health professionals yes/no & $380(89.6) / 116(82.3)$ & $44(10.4) / 25(17.7)$ & $\mathrm{p}=0.021$ \\
\hline Knowledge from the press, TV and newspapers, books yes/no & $327(88.4) / 169(86.7)$ & $43(11.6) / 26(13.3)$ & $\mathrm{p}=0.555$ \\
\hline Knowledge from family and friends yes/no & $162(88.5) / 334(87.4)$ & $21(11.5) / 48(12.6)$ & $\mathrm{p}=0.711$ \\
\hline Knowledge from the Internet yes/no & $121(91.7) / 375(86.6)$ & $11(8.3) / 58(13.4)$ & $\mathrm{P}=0.120$ \\
\hline Obesity no/yes & $307(86.7) / 189(89.6)$ & $47(13.3) / 22(10.4)$ & $\mathrm{p}=0.317$ \\
\hline Current smoker yes/no & $74(82.2) / 422(88.8)$ & $16(17.8) / 53(11.2)$ & $\mathrm{p}=0.079$ \\
\hline
\end{tabular}

Data is presented as $\mathrm{n}(\%) ;{ }^{*}$ not including people who replied "I have no opinion"; ${ }^{* *}$ combination of high and moderate impact; ${ }^{* * *}$ combination of low and no impact 
Table XIII. Factors affecting knowledge of the influence of stressful lifestyle on the development of HT

\begin{tabular}{|l|c|c|c|}
\hline $\mathbf{n}=\mathbf{5 9 1 *}$ & Significant impact** & No impact*** & $\mathbf{p}$ \\
\hline Female/male & $321(96.1) / 237(92.2)$ & $13(3.9) / 20(7.8)$ & $\mathrm{p}=0.041$ \\
\hline Age $<49$ years/> 49 years & $109(94.0) / 449(94.5)$ & $7(6.0) / 26(5.5)$ & $\mathrm{p}=0.814$ \\
\hline Married/single & $419(94.6) / 139(93.9)$ & $24(5.4) / 9(6.1)$ & $\mathrm{P}=0.761$ \\
\hline Rural/urban residence & $263(92.9) / 295(95.8)$ & $20(7.1) / 13(4.2)$ & $\mathrm{p}=0.132$ \\
\hline Primary education or vocational/secondary or higher & $230(92.7) / 328(95.6)$ & $18(7.3) / 15(4.4)$ & $\mathrm{p}=0.132$ \\
\hline Professionally active yes/no & $233(94.3) / 325(94.5)$ & $14(5.7) / 19(5.5)$ & $\mathrm{p}=0.940$ \\
\hline Knowledge from health professionals yes/no & $423(96.1) / 135(89.4)$ & $17(3.9) / 16(10.6)$ & $\mathrm{p}=0.002$ \\
\hline Knowledge from the press, TV and newspapers, books yes/no & $361(93.5) / 197(96.1)$ & $25(6.5) / 8(3.9)$ & $\mathrm{p}=0.195$ \\
\hline Knowledge from family and friends yes/no & $185(95.4) / 373(93.9)$ & $9(4.6) / 24(6.1)$ & $\mathrm{p}=0.484$ \\
\hline Knowledge from the Internet yes/no & $125(94.0) / 433(94.5)$ & $8(6.0) / 25(5.5)$ & $\mathrm{p}=0.806$ \\
\hline Obesity no/yes & $339(94.2) / 219(94.8)$ & $21(5.8) / 12(5.2)$ & $\mathrm{p}=0.741$ \\
\hline Current smoker yes/no & $85(92.4) / 473(94.8)$ & $7(7.6) / 26(5.2)$ & $\mathrm{p}=0.357$ \\
\hline
\end{tabular}

Data is presented as $n(\%) ;{ }^{*}$ not including people who replied "I have no opinion"; ${ }^{* *}$ combination of high and moderate impact; ${ }^{* * *}$ combination of low and no impact

ination of the exacerbating factors, which in turn requires them to have the appropriate knowledge.

Several decades ago, the adverse effects of alcohol on the RR in patients treated with HT were confirmed. Limiting alcohol intake is associated with reduced need for antihypertensive drugs and better control of RR $[4,5]$. Unfortunately, false information or lack of knowledge in this area was observed in about $1 / 3$ of respondents, $16 \%$ and $15 \%$, respectively. In fact, people who got it from the Internet were more likely to have false information. Therefore, it seems appropriate to spread knowledge more widely, especially on websites.

Drinking coffee systematically does not affect the development of HT, but it can contribute to reducing mortality due to cardiovascular disease, lung diseases, diabetes, traumas and infections [17, 21]. Unfortunately, more than $55 \%$ of people in the study had incorrect knowledge of the subject. This phenomenon especially affected patients who received information from family and friends, the group that seems to be the most difficult to reach in social campaigns.

So far no direct connection has been proven between smoking and HT development, despite its association with mortality due to cardiovascular disease $[15,16]$. In the study group, nearly three quarters believed that nicotine was associated with hypertension. This situation was more frequent in people who currently smoke cigarettes and seek information from health professionals. Perhaps HT patients are informed in health care facilities about the effects of smoking on the increased risk of cardiovascular disease and therefore they answered incorrectly more often about its effect on the RR.
The effect of consuming excessive amounts of salt on HT development has been frequently proven. Even the value of the decrease in RR when using a low sodium diet was estimated [5]. The respondents presented relatively good knowledge about the effect of salt intake on HT. A little over 7\% gave a wrong answer and less than $4.5 \%$ did not have any opinion about this connection. Patients who did not receive information from health professionals were reported to have incorrect information slightly more often.

So far, many studies have examined the effects of individual dietary components on cardiovascular morbidity. Recent studies have shown that fat reduction alone did not affect HT development [20]. Of the respondents, $88 \%$ had false knowledge about the effect of high intake of fat on HT development, and nearly $7 \%$ did not have opinion about this effect. This was the worst result in the study. Women and patients who were informed by health professionals responded incorrectly more often. Likewise, obese patients demonstrated a lower level of knowledge [23].

The general opinion is that irregular eating can lead to obesity and many diseases associated with it, i.e. HT. No evidence has yet been found in clinical trials of the direct effect of irregular meals on the development of HT. In this study, incorrect knowledge about the effects of irregular eating on HT development was reported by over $46 \%$ of respondents, and more than $24 \%$ did not comment on this. More often, patients who received information from health professionals, family and friends, and the Internet were misinformed.

Changing diet can significantly reduce HT development. In the studies published in 1997 and 2000, the effects of a diet containing fibre, vegetables and 
skimmed dairy products on the reduction of RR have been confirmed. Nearly $30 \%$ of the respondents did not comment on the impact of the aforementioned diet on HT development and nearly $28 \%$ had misleading information. Slightly more often, people who did not receive information from health professionals answered incorrectly. The above data show poor knowledge of the correct eating habits irrespective of sociodemographic characteristics of patients.

One element of a healthy lifestyle that can reduce $\mathrm{RR}$ and therefore antihypertensive drugs intake is to lose weight in overweight or obese patients [9]. Only $4.4 \%$ of respondents mistakenly answered the question about the connection between HT and excess weight. This was the best result in the poll. People living in rural areas, as compared to urban dwellers, had misleading information more often.

Until now, there is no convincing evidence for the connection between higher cholesterol levels and other lipid disorders on the development of HT. Despite this, almost $88 \%$ of respondents gave an incorrect answer about the connection between the two disorders. This was one of the two worst results in the study. Especially those who obtained information from family and friends had incorrect knowledge.

Regular exercise exerts a positive influence on cardiovascular risk factors including $\mathrm{RR}$, and in patients with HT it allows the reduction of antihypertensives $[5,10]$. In the surveyed population, more than $11 \%$ of the respondents had a false belief about the insignificance of low physical activity on the development of HT, and almost $8 \%$ had no opinion on it. Especially those living in the countryside, with primary or vocational education, not receiving information from healthcare professionals, gave an incorrect answer.

A cause-and-effect relationship between stressful lifestyle and HT and ischaemic heart disease has been proven [12]. As many as $91 \%$ of respondents gave the correct answer, which was the second result in this study. Incorrect knowledge concerned especially men and those not receiving information from health professionals.

\section{Conclusions}

1. Incorrect knowledge of factors affecting the development of HT concerned in particular the consumption of excessive amounts of fat, raised cholesterol, nicotine/smoking and drinking coffee.

2. There were no common features of people who answered the questions incorrectly.
3. Results of the study indicate the need to educate people with HT on the factors influencing the development of this disease.

\section{References}

1. Kearney PM, Whelton M, Reynolds K, et al. Global burden of hypertension: analysis of worldwide data. Lancet. 2005; 365(9455): 217-223, doi: 10.1016/S0140-6736(05)17741-1, indexed in Pubmed: 15652604

2. Zdrojewski $€$, Zdrojewski T, Rutkowski M, et al. Prevalence and control of cardiovascular risk factors in Poland. Assumptions and objectives of the NATPOL 2011 Survey. Kardiol Pol. 2013; 71(4): 381-392, doi: 10.5603/KP.2013.0066, indexed in Pubmed: 23788344.

3. Lalonde M. A New Perspective on the Health of Canadians, a working document. : 1974 .

4. Puddey IB, Beilin LJ, Vandongen R. Regular alcohol use raises blood pressure in treated hypertensive subjects. A randomised controlled trial. Lancet. 1987; 1(8534): 647-651, indexed in Pubmed: 2882082.

5. Dickinson HO, Mason JM, Nicolson DJ, et al. Lifestyle interventions to reduce raised blood pressure: a systematic review of randomized controlled trials. J Hypertens. 2006; 24(2): 215-233, doi: 10.1097/01.hjh.0000199800.72563.26, indexed in Pubmed: 16508562.

6. Rupp H, Brilla CG, Maisch B. [Hypertension and alcohol: central and peripheral mechanisms]. Herz. 1996; 21(4): 258-264, indexed in Pubmed: 8805006.

7. Reisin E. Sodium and obesity in the pathogenesis of hypertension. Am J Hypertens. 1990; 3(2): 164-167, indexed in Pubmed: 2155002.

8. Wofford MR, Hall JE. Pathophysiology and treatment of obesity hypertension. Curr Pharm Des. 2004; 10(29): 3621-3637, indexed in Pubmed: 15579059.

9. Neter JE, Stam BE, Kok FJ, et al. Influence of weight reduction on blood pressure: a meta-analysis of randomized controlled trials. Hypertension. 2003; 42(5): 878-884, doi: 10.1161/01. HYP.0000094221.86888.AE, indexed in Pubmed: 12975389.

10. Cornelissen VA, Fagard RH. Effects of endurance training on blood pressure, blood pressure-regulating mechanisms, and cardiovascular risk factors. Hypertension. 2005; 46(4): 667-675, doi: 10.1161/01. HYP.0000184225.05629.51, indexed in Pubmed: 16157788.

11. Szczepańska-Sadowska E. Sikora M. Patofizjologia nadciśnienia tętniczego. Więcek A. Januszewicz A. Szczepańska-Sadowska E Prejbisz A. Hipertensjologia, patogeneza, diagnostyka i leczenie nadciśnienia tętniczego. Medycyna Praktyczna, Kraków. 2011; 2: 23-175.

12. Pickering TG. Mental stress as a causal factor in the development of hypertension and cardiovascular disease. Curr Hypertens Rep. 2001; 3(3): 249-254, indexed in Pubmed: 11353576.

13. Omvik P. How smoking affects blood pressure. Blood Pressure. 2009; 5(2): 71-77, doi: 10.3109/08037059609062111.

14. James JE. Critical review of dietary caffeine and blood pressure: a relationship that should be taken more seriously. Psychosom Med. 2004; 66(1): 63-71, indexed in Pubmed: 14747639.

15. Doll R, Peto R, Boreham J, et al. Mortality in relation to smoking: 40 years' observations on male British doctors. BMJ. 1994; 309(6959): 901-911, indexed in Pubmed: 7755693.

16. Primatesta P, Falaschetti E, Gupta S, et al. Association between smoking and blood pressure: evidence from the health survey for England. Hypertension. 2001;37(2): 187-193, indexed in Pubmed: 11230269 .

17. Steffen M, Kuhle C, Hensrud D, et al. The effect of coffee consumption on blood pressure and the development of hypertension: a systematic review and meta-analysis. J Hypertens. 2012; 30(12): 2245-2254, doi: 10.1097/HJH.0b013e3283588d73, indexed in Pubmed: 23032138.

18. Appel LJ, Moore TJ, Obarzanek E, et al. A clinical trial of the effects of dietary patterns on blood pressure. DASH Collaborative Research 
Group. N Engl J Med. 1997; 336(16): 1117-1124, doi: 10.1056/ NEJM199704173361601, indexed in Pubmed: 9099655.

19. Conlin PR, Chow D, Miller ER, et al. The effect of dietary patterns on blood pressure control in hypertensive patients: results from the Dietary Approaches to Stop Hypertension (DASH) trial. Am J Hypertens. 2000; 13(9): 949-955, indexed in Pubmed: 10981543.

20. Allison MA, Aragaki AK, Ray RM, et al. A Randomized Trial of a Low-Fat Diet Intervention on Blood Pressure and Hypertension: Tertiary Analysis of the WHI Dietary Modification Trial. Am J Hypertens. 2016; 29(8): 959-968, doi: 10.1093/ajh/hpv196, indexed in Pubmed: 26708006.

21. Freedman ND, Park Y, Abnet CC, et al. Association of coffee drinking with total and cause-specific mortality. N Engl J Med. 2012; 366(20): 1891-1904, doi: 10.1056/NEJMoa1112010, indexed in Pubmed: 22591295.

22. Gać P, Poręba R, Poręba M, et al. Znajomość przyczyn nadciśnienia tętniczego i skutków jego nieleczenia w losowej grupie studentów wybranych miast Polski. Nadciśnienie Tętnicze. 2013; 17(5):377-383.

23. Bronkowska M, Martynowicz H, Żmich K, et al. Wybrane elementy stylu życia oraz wiedza żywieniowa otyłych osób z rozpoznanym nadciśnieniem tętniczym. Nadciśnienie Tętnicze. 2009; 13(4): 266-274.
24. Georgiopoulou VV, Kalogeropoulos AP, Raggi P, et al. Prevention, diagnosis, and treatment of hypertensive heart disease. Cardiol Clin. 2010; 28(4): 675-691, doi: 10.1016/j.ccl.2010.07.005, indexed in Pubmed: 20937450.

25. Erbel R, Aboyans V, Boileau C, et al. Wytyczne ESC dotyczące rozpoznawania i leczenia chorób aorty w 2014 roku. Kardiologia Polska. 2014; 72(12): 1169-1252, doi: 10.5603/kp.2014.0225.

26. Montalescot G, Sechtem U, Achenbach S, et al. Wytyczne ESC dotyczące postępowania w stabilnej chorobie wieńcowej w 2013 roku. Kard Pol. 2013; 71(9): 224-317.

27. Mancia G, Fagard R, Narkiewicz K, et al. Wytyczne ESH/ESC dotyczące postępowania w nadciśnieniu tętniczym w 2013 roku. Kard Pol. 2013; 71(3): 60-64.

28. Kirchhof P, Benussi S, Kotecha D, et al. Wytyczne ESC dotyczące leczenia migotania przedsionków w 2016 roku, opracowane we współpracy z EACTS. Kardiologia Polska. 2016; 74(12): 1359-1469, doi: $10.5603 / \mathrm{kp} .2016 .0172$.

29. Szczepańska-Sadowska E. Sikora M. Nadciśnienie tętnicze pierwotne. Więcek A., Januszewicz A., Szczepańska-Sadowska E., Prejbisz A. Hipertensjologia, patogeneza, diagnostyka i leczenie nadciśnienia tętniczego. Medycyna Praktyczna, Kraków. 2011; 2: 291-394. 